\title{
An evaluation of mechanically co-ordinated legged locomotion (The Iron Mule Train revisited) D.J. Todd
}

\author{
Department of Mechanical Engineering, University of Edinburgh, King's Buildings, Mayfield Road, Edinburgh \\ EH93JL (UK)
}

(Received in final form: November 3, 1990)

\section{SUMMARY}

An eight-legged walking machine with purely mechanical control of leg motion has been built, based on an old design by Space-General Corporation. The paper describes its mechanical design, and experiments with it on a variety of surfaces.

KEYWORDS: Legged locomotion; Mechanical co-ordination; Design.

\section{INTRODUCTION}

More than twenty years ago, the Space-General Corporation (SGC) developed several prototypes of a walking vehicle whose leg movements were generated purely mechanically. This type of machine was considered for planetary exploration, as a walking chair for the disabled, and for military transport, in which role it was sometimes referred to as the "Iron Mule Train".

The company reported various successful experiments ${ }^{1}$ but the approach was never taken further, and since then purely mechanical walkers have been eclipsed by those with computer-controlled, independently actuated joints, which for most purposes confer critical advantages.

Nevertheless, the idea has never quite died, and people still try to build legged robots which use mechanical linkages to reduce the number of independently actuated joints. Our own reasons for an excursion into purely mechanical walkers were several. First, we needed a small walking machine for experiments on the interaction of legs with soil and vegetation, and a purely mechanical design would allow it to be built in a few months. Second, the approach seemed worth reexamining for the walking chair application, as a mechanical walker might be cheaper, and simpler to maintain, than a computer-controlled, fully adaptable one. Finally, there is a growing market for 'robotic' devices for museums, exhibitions, leisure parks and so on. For such applications the terrain can often be landscaped to limit the difficulty of locomotion, and then the mechanical walking base may be a feasible element of various robotic machines.

\section{MECHANICAL DESIGN}

The machine is based on the Space General design, with modifications to make certain components easier to manufacture. It has eight legs, a number which allows it to be divided into two halves, each with its own motor, so it can be skid-steered like a tracked vehicle. Of the four legs on each side, one front and one rear leg are in the support phase while the other pair are being protracted. Each leg is operated by a pair of cams, one cam for the horizontal component of foot motion and one for the vertical. In the SGC design (Figure 1a) these cams were located on the same shaft (in contrast to the new design, shown in Figure 1b). Both cams, which have a complex lobed shape, have to be grooved so the cam can pull the follower as well as push it. In our design, the lift cam does not usually have to pull the follower against any great force, and in the initial version is a simple flat plate. A small force is still necessary to overcome the weight of the legs; this is provided by a tension spring. The horizontal-motion cam is still grooved, but for the first version has been made circular for ease of manufacture. These features are shown in Figure 2. The dimensions of the vehicle are given in the Appendix.

The cam profiles were chosen to generate a roughly $D$-shaped foot trajectory so each foot travels in a straight horizontal line during the support phase; an example of a foot trajectory is shown in Figure 3. The front and back leg-pairs in each side of the machine share a vertical-motion camshaft having four cams, but have separate shafts, with two cams each, for the horizontal component of motion (Figure 1b). The three shafts are synchronized and powered by a common roller chain driven by a geared $24 \mathrm{~V} \mathrm{DC}, 20 \mathrm{~W}$ motor.

In the SPG machine, the two halves were rigidly connected. This has the result that on rough ground only three of the four nominally supporting legs make contact with the ground. In the present machine the two halves are connected by a single lateral shaft which allows rotation about a pitch axis. The machine in effect becomes two narrow quadrupeds walking side by side and leaning on each other for mutual support. Each can follow local variations in ground height and the load is more evenly distributed. However, for stair climbing this creates problems, so the two halves are secured rigidly together.

\section{CONTROL}

So far the machine has always been operated by a person carrying a switch box or pendant connected to the 


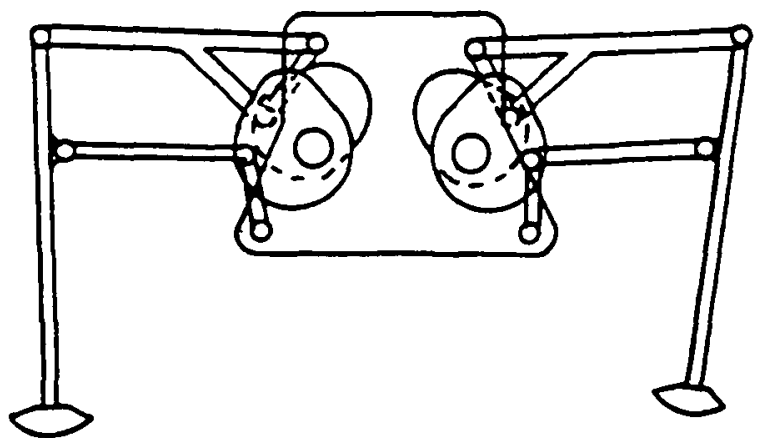

(a)

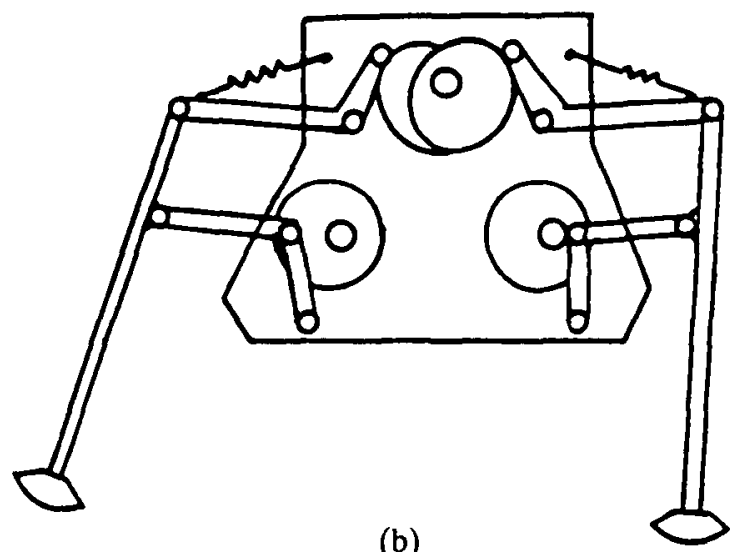

(b)

Fig. 1. Comparison of original and new cam arangements. Only two of the four legs in one side of the machine are shown. (a) SPG design. The lift cam is on the same shaft as the horizontal movement cam. (b) Design with separate camshaft for lift.

vehicle by a cable. The pendant allows the user to switch each motor on or off, and reverse it, independently. It is possible to make small corrections to the octopod's course by stopping one side for a moment. The machine's smoothness of progression would benefit from servo velocity control of the motors; this has not yet been tried.
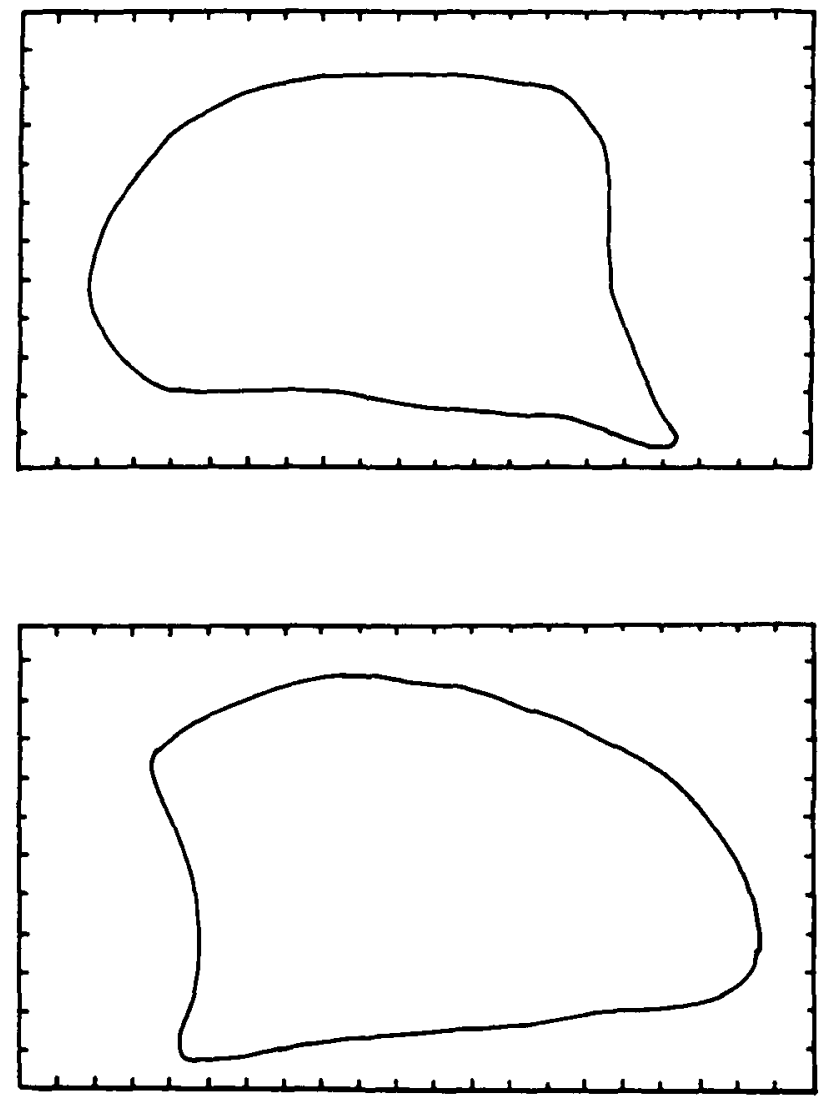

Fig. 3. Trajectory, relative to the vehicle, of a leading and a trailing foot on the same side. The tick marks are at centimetre intervals; the origin is arbitrary.

\section{EXPERIMENTS}

\subsection{Smooth floor walking}

On level ground the vehicle walks at about $0.13 \mathrm{~m} / \mathrm{s}$ when the battery voltage is $24 \mathrm{~V}$. In early tests a smaller-gear ratio was tried and it walked twice as fast. The present motors are rather small and with larger ones

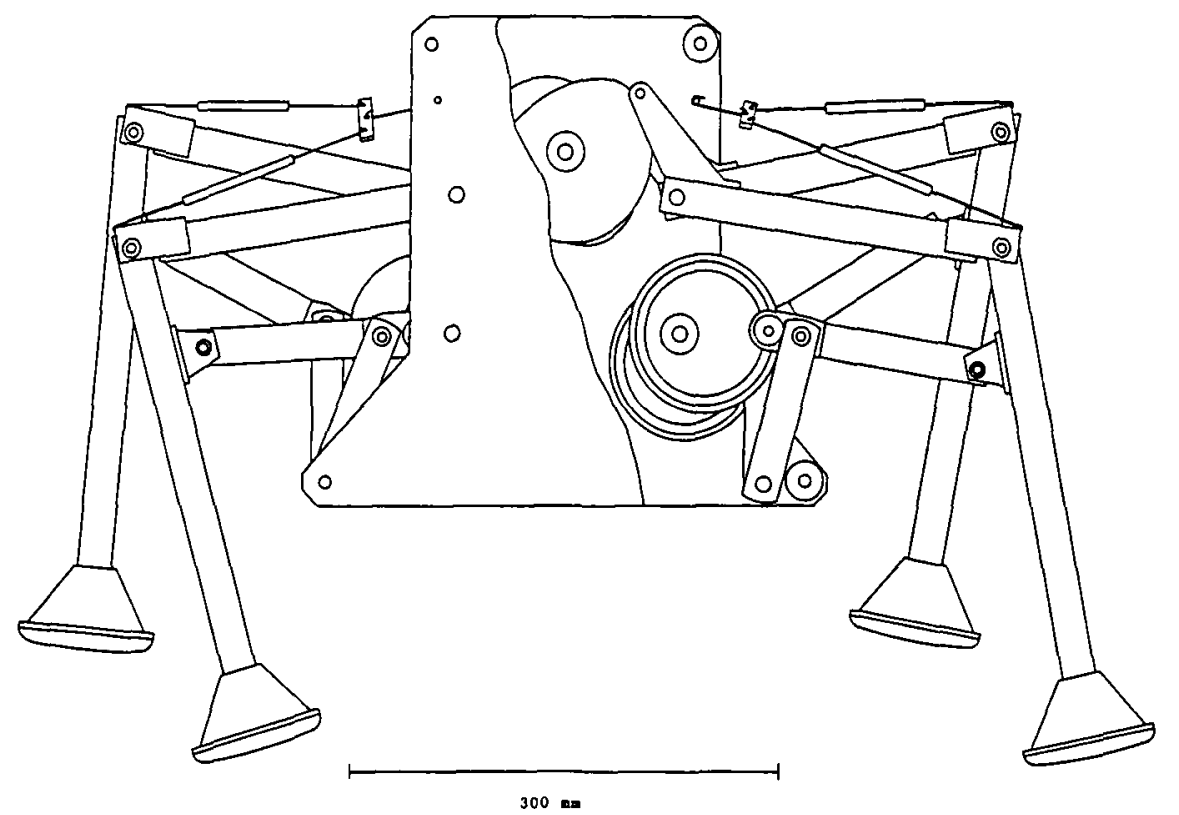

Fig. 2. Cutaway drawing of one half of the octopod. The roller chain linking the three shafts and the motor is not shown. The two tension springs of each pair are joined together at their inboard end by a yoke whose pivoting minimizes the length change undergone by each spring. 
there would seem no obstacle to attaining speeds up to about $0.5 \mathrm{~m} / \mathrm{s}$; above this inertial forces and shock loading would create problems.

Turning on the spot (by reversing one motor) looks rather erratic since the precise behaviour depends on the local friction characteristics of each foot-ground contact point, but is reasonably effective. Larger radius turns can be achieved more smoothly, by slowing one side rather than reversing it.

\subsection{Soil and Vegetation}

For some applications of autonomous mobile robots, vegetation may be the factor limiting mobility. One aspect of this is the machine's size relative to the spacing between immovable objects such as trees, but another is the way in which smaller plants (and roots and low branches of larger ones) impede the legs.

One observation (rather obvious in retrospect) was that unless the legs are shaped suitably, the feet tend to catch on vegetation. Many legged robots have rather large feet, either to limit ground pressure or to accommodate joints, sensors or actuators. As first built, the octopod had feet $70 \mathrm{~mm}$ long by $45 \mathrm{~mm}$ wide, whereas the legs are $25 \mathrm{~mm}$ square (Figure 4a). Such feet are ideal for catching on roots and digging into soft surfaces, and on testing on a bed planted with a creeping ground-cover plant having long, near-horizontal woody stems a few inches above ground (Figure 5), the machine

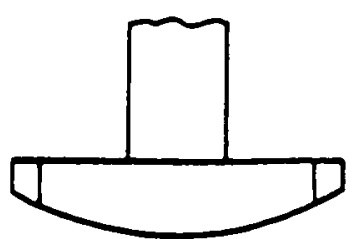

(a)

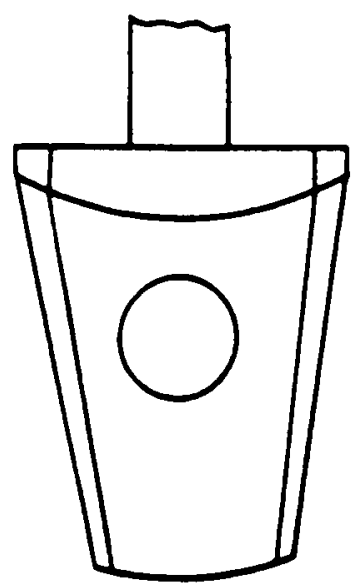

(b)

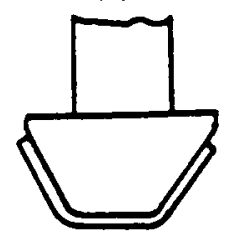

(c)

Fig. 4. Three designs of foot. (a) Original foot, (b) Foot with stilt to prevent catching on vegetation, (c) Small foot with rubber sole for stair climbing. repeatedly caught its feet and halted. This problem was overcome by fitting a 'stilt' in the form of a truncated pyramid to each foot (Figure 3b). The taper was such that a raised foot swinging forward and meeting an obstruction would tend to slide up and over it.

It is interesting to note that vertebrates often have large feet without being much troubled by this problem. They solve it largely by using an ankle pitch joint which allows the foot to swing down and back at will. The knee sometimes fulfils a similar role. A second factor is being able to anticipate possible foot catching and raise the foot to an increased height during protraction. Personal experience shows that neither solution is always effective.

The machine has also been operated on bare earth, sand and gravel. Although we have done no comparison with a wheeled vehicle of similar size, we get the impression that the walker's interaction with the ground is efficient: the feet tend to sink in a short way (about an inch in soft builders' sand) then provide a good grip for traction. The machine will climb a slope of about $30^{\circ}$ in sand (Figure $5 \mathrm{~b}$ ). The main problem in climbing a slope is that unless continuous corrections are made, the vehicle tends to veer off across the slope.

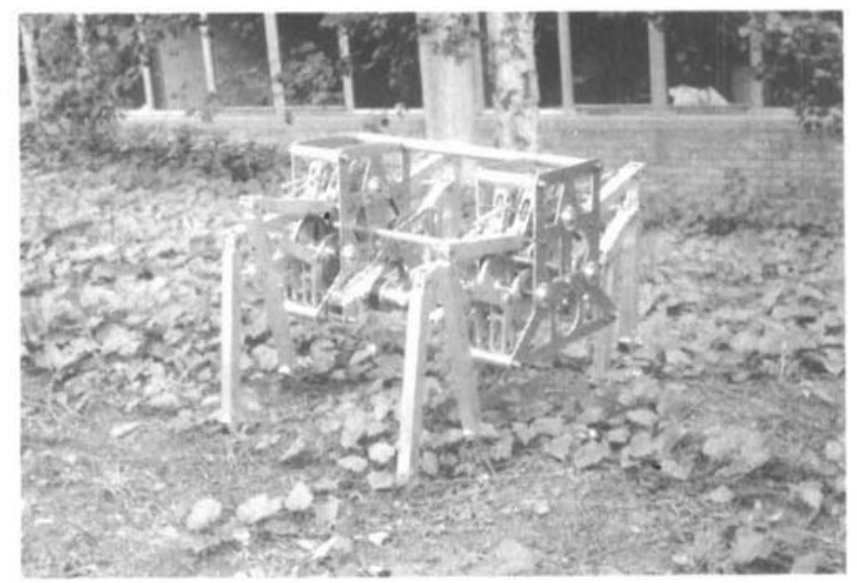

(a)

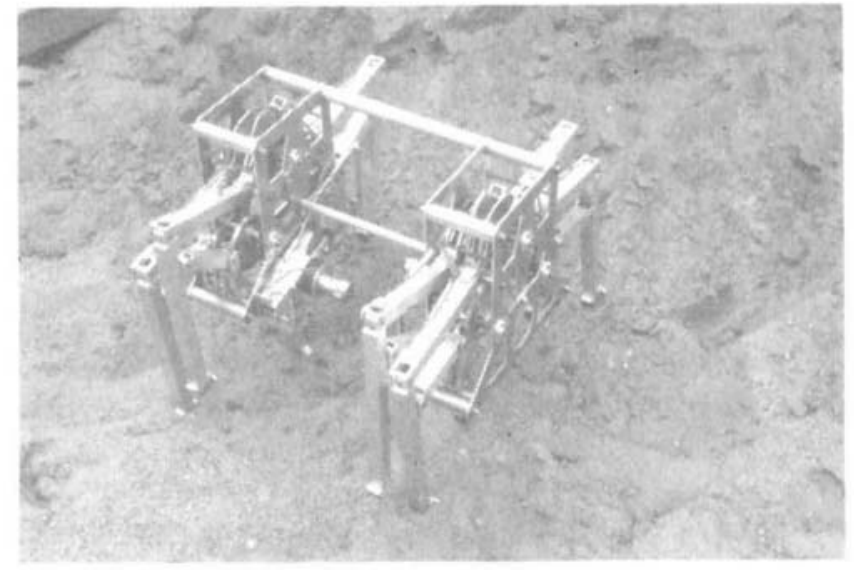

(b)

Fig. 5. Octopod on three kinds of terrain. For the terrains of (a) and (b) it was fitted with larger feet, and the upper tie bar between the two halves was disconnected. (a) Soil with creeping ground-cover plants, (b) Octopod in builders' sand, (c) Stair climbing. 


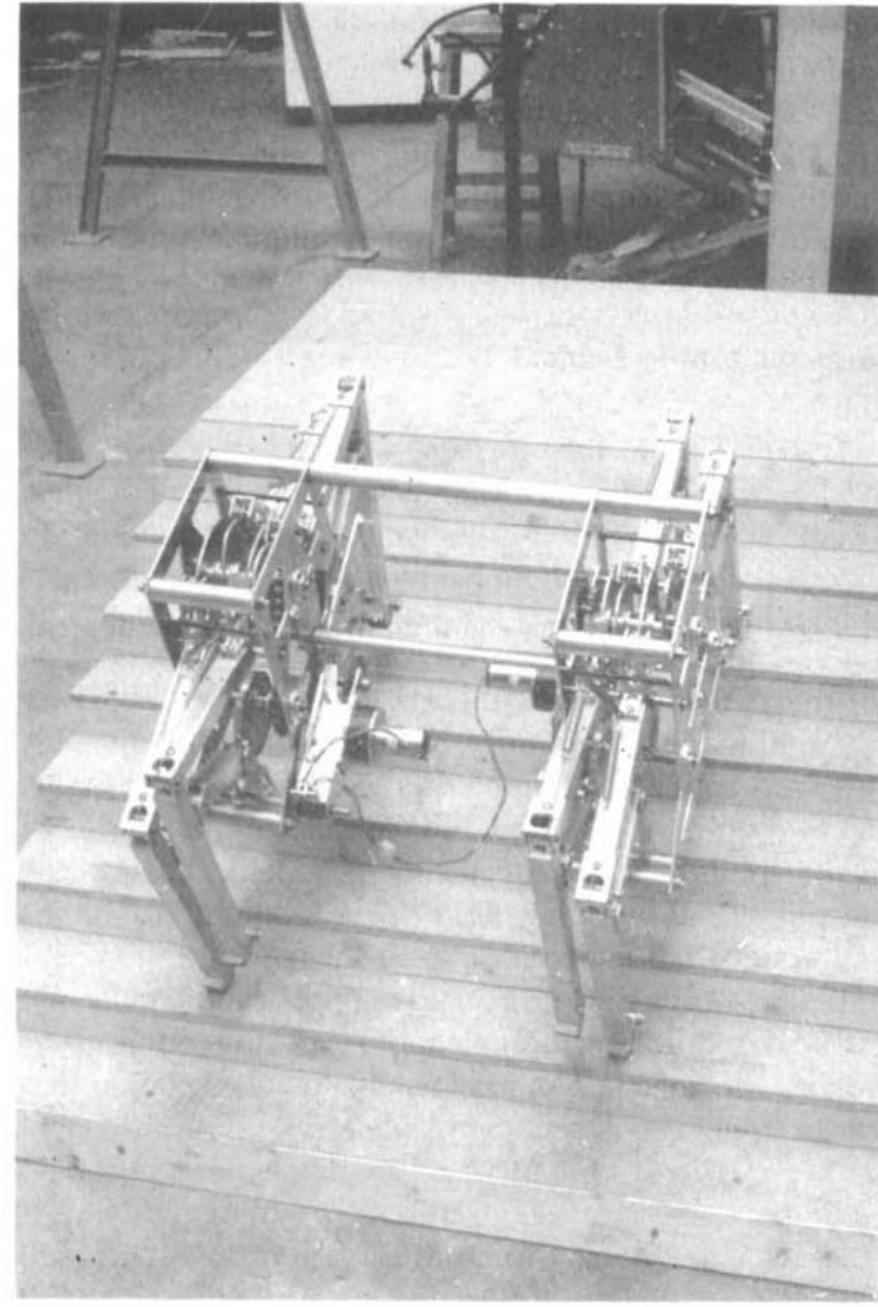

(c)

Fig. 5. (continued)

\subsection{Stairs}

Since a machine of this kind cannot select its footholds, one would expect that climbing stairs would be rather erratic, the feet sometimes landing right on the edge of a stair tread and slipping off. Yet Space General claimed their prototype was very successful at stair climbing, so we were interested in how our machine would perform. Since it is too small to climb real stairs, a flight of eight scaled-down ones was built, with treads $125 \mathrm{~mm}$ wide and risers $60 \mathrm{~mm}$ high; the vehicle lifts its feet 70 or $80 \mathrm{~mm}$.

With the original feet, and also with the stilts, the octopod nearly always gets stuck, a protracting foot catching on a riser of the stair. Although the feet are raised well above the height of a step, and although they leave the ground almost vertically relative to the vehicle, when forward motion is taken into account a foot leaves the ground with a significant forward component and often has not gone far when it meets a step. The springs which hold the cam followers onto the cams are not always strong enough to overcome the resulting frictional force.

The problem was to a large extent solved by fitting a third type of foot, with a small roller on the 'toe' (Figures 3c, 5c). However, a more certain solution is to go back to grooved cams as used on the SGC machines so there is a positive force lifting the feet, rather than just springs.

With the rollers, the octopod does generally succeed in climbing its stairs. It is often necessary to make steering corrections by momentarily stopping one or other motor if that side gets ahead (usually because a foot has caught briefly on a step). The motion is, as predicted, often rather erratic, although if no foot happens to get caught or slip off an edge the machine can give an illusion of sure-footed and positive progress. On balance, it seems unlikely that many disabled people would want to entrust themselves to such a device on a steep flight of stairs.

\subsection{Smooth Floor with Obstacles}

The octopod was also tested on a smooth floor randomly scattered with objects up to about $50 \mathrm{~mm}$ high. The objects were: fixed angle-iron supports for a platform; small bricks; lengths of wood; and a length of $50 \mathrm{~mm}$ diameter steel pipe.

Its progression is stable and reasonably smooth, considering that it has no way of detecting obstacles. The feet either land between the objects, sometimes pushing them out of the way, or else land on them and use them for support. The two sides of the body make independent pitch excursions of up to about $10^{\circ}$.

\section{CONCLUSIONS}

We now have a small legged vehicle which is available for further experiments on soil mechanics and is useful for teaching and demonstrations. It illustrates for students and visitors the strengths and limitations of a non-adaptive walking machine. It could be the foundation of a variety of student projects, such as fitting it with on-board computing and obstacle sensing, or trying hybrid designs in which, say, the vertical motion is still driven by cams but horizontal motion uses an individual actuator for each leg, allowing control of foot placement.

We do not pretend that this machine is a technical advance on what has gone before, but on balance building it has been worthwhile.

\section{References}

1. R.A. Morrison, "Iron Mule Train" Cornell Aeronatical Laboratory/International Society for Terrain Vehicle Systems Off Road Mobility Research Symposium, Washington D.C. (June, 1968) pp. 383-400.

$\begin{array}{lc}\text { APPENDIX - DIMENSIONS } \\ \text { Length of body } & 0.37 \mathrm{~m} \\ \text { Length overall } & 0.60 \mathrm{~m} \\ \text { Width } & 0.56 \mathrm{~m} \\ \text { Overall height } & 0.50 \mathrm{~m} \\ \text { Height to bottom of body } & 0.17 \mathrm{~m} \\ \text { Horizontal foot stroke } & 0.15 \mathrm{~m} \\ \text { Vertical foot stroke } & 0.08 \mathrm{~m} \\ \text { Weight } & 13 \mathrm{~kg} \\ \text { Typical walking speed } & 0.13 \mathrm{~m} / \mathrm{s}\end{array}$

